\title{
Phaeochromocytoma presenting as Takotsubo cardiomyopathy
}

\author{
Authors: Oluwatosin Kayode, ${ }^{A}$ Paul Underwood, ${ }^{B}$ Ravi Dissanayake, ${ }^{A}$ David Scott-Coombes ${ }^{C}$ and \\ Subramaniam Nagasayi ${ }^{\mathrm{D}}$
}

We report a case of a 72-year-old woman who presented with ST-elevation myocardial infarction (STEMI). However, coronary angiography showed unobstructed arteries while echocardiography (ECHO) showed severe left ventricular (LV) apical hypokinesia with ejection fraction (EF) of $25-30 \%$. Seven months later she presented with a transient ischaemic attack and a repeat $\mathrm{ECHO}$ showed a normal EF.

A few months later, she was diagnosed with breast cancer and as part of staging procedure, an incidental left adrenal mass was identified. This was biochemically confirmed as phaeochromocytoma (PY) and she underwent laparoscopic adrenalectomy.

PY is a rare catecholamine secreting tumour arising from adrenomedullary chromaffin cells. Excessive catecholamineinduced stimulation can present as transient, reversible cardiomyopathy similar to Takotsubo cardiomyopathy and cerebrovascular events. The diagnosis of PY is often delayed but it is important to recognize PY as a cause of reversible cardiomyopathy. Early intervention is essential to improve mortality from cardiovascular and cerebrovascular complications.

KEYWORDS: Acute myocardial infarction, transient ischemic attack, Takotsubo cardiomyopathy, phaeochromocytoma

DOI: $10.7861 /$ clinmed.2019-0494

\section{Case presentation}

A 72-year-old woman presented with typical cardiac chest pain lasting an hour. She had intermittent history of palpitations, headache, dizziness, sweatiness and weight loss over the last 18 months. Her past medical history included hypertension and type 2 diabetes. She had never smoked and consumed alcohol occasionally. Her mother had ischaemic heart disease. She denied any recent stressful life events. Her medications included

Authors: ${ }^{\text {A }}$ specialty doctor in endocrinology/diabetes, Withybush General Hospital, Haverfordwest, UK; ${ }^{B}$ consultant in endocrinology/ diabetes, Withybush General Hospital, Haverfordwest, UK;

${ }^{C}$ consultant endocrine surgeon, University Hospital of Wales

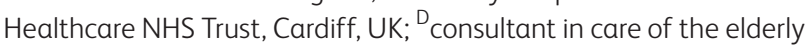
medicine, Withybush General Hospital, Haverfordwest, UK ramipril, $5 \mathrm{mg}$ once daily, and metformin, $500 \mathrm{mg}$ twice daily. On examination in the emergency department, she was afebrile, oxygen saturations at $99 \%$ on air, respiratory rate was 18 breaths per minute, pulse was 64 beats per minute and blood pressure was $158 / 85 \mathrm{mmHg}$. Systemic examination revealed normal cardiorespiratory and abdominal findings.

Blood results were haemoglobin of $130 \mathrm{~g} / \mathrm{L}$, glycated haemoglobin (HbA1c) of $60 \mathrm{mmol} / \mathrm{mol}$, mean corpuscular volume of $90 \mathrm{fL}$, C-reactive protein of $<5 \mathrm{mg} / \mathrm{L}$, creatinine of $85 \mu \mathrm{mol} / \mathrm{L}$, urea of $6 \mathrm{mmol} / \mathrm{L}$ and troponin of $1.372 \mu \mathrm{g} / \mathrm{L}$. Chest X-ray showed no evidence of pulmonary congestion or effusion. Electrocardiography (ECG) showed ST segment elevation myocardial infarction (STEMI), deep T wave inversion and QT interval prolongation. She underwent coronary intervention in a tertiary cardiac unit.

\section{Diagnosis}

Common causes of life-threatening cardiovascular chest pain include acute coronary syndrome (ACS), acute myocarditis, aortic dissection and acute pulmonary embolism. The most common cause is ACS due to plaque rupture and vessel occlusion. In presence of angiographically normal coronary arteries, one has to consider coronary vasospasm, vasculitis, myocarditis or type 2 myocardial infarction (supply vs demand).

\section{Initial management and prognosis}

Since our patient had typical symptoms, diagnostic ECG changes with very high cardiac markers, ACS was the most likely diagnosis. Coronary angiography, however, showed unobstructed normal arteries. Echocardiography (ECHO) showed apical hypokinesia, preserved basal segments and severe left ventricular (LV) systolic dysfunction with estimated ejection fraction (EF) of 25-30\%. She was discharged home on secondary prevention with aspirin, bisoprolol, rosuvastatin with plans for local follow-up and repeat $\mathrm{ECHO}$ in 3 months to reassess LV function.

\section{Case progression and outcome}

After 7 months, she experienced a transient ischaemic attack with expressive dysphasia. A 12-lead ECG showed complete resolution of her previous ECG changes. Magnetic resonance imaging (MRI) of the brain and carotid Dopplers were normal. ECHO was repeated and showed complete resolution of previous LV wall changes and function, hence ticagrelor and eplerenone were stopped. 
Unfortunately, 4 months later, she was diagnosed with intraductal breast cancer. A staging computed tomography (CT) of the abdomen and pelvis identified a left adrenal mass and this prompted an endocrinology consult. Twenty-four hour urinary metadrenaline were significantly elevated and phaeochromocytoma (PY) was diagnosed. Preoperatively, her blood pressure was controlled with phenoxybenzamine followed by beta blockers. She underwent laparoscopic adrenalectomy and, postoperatively, phenoxybenzamine was stopped. Her $\mathrm{HbA1}$ c was back within normal range at $39 \mathrm{mmol} / \mathrm{mol}$ and blood pressure stable on bisoprolol. She awaits wire-guided wide local excision of breast lesion.

\section{Discussion}

$\mathrm{PY}$ is a rare catecholamine secreting tumour arising from adrenomedullary chromaffin cells which typically manifests with sustained or paroxysmal clinical features including hypertension, palpitations, tachycardia, headache, and diaphoresis. Excessive catecholamine-induced stimulation of cardiac myocytes can present as transient, reversible Takotsubo cardiomyopathy (TC). ${ }^{1}$

Patients with PY may show typical cardiovascular,

cerebrovascular, and renal effects of prolonged hypertension, such as congestive heart failure with pulmonary oedema, cardiogenic shock and cardiac arrhythmias.

TC, also known as apical ballooning syndrome or broken-heart syndrome, is a recognised but uncommon occurrence with PY presents as ACS characterised by severe left ventricular (LV) dysfunction that typically recovers spontaneously within days or weeks. ${ }^{2}$ It accounts for $2.0 \%$ of ST-segment elevation infarct and most often occurs in postmenopausal women after intense emotional or physical stress. The proposed mechanisms of cardiac dysfunction include direct toxic effects of catecholamine by-products on the myocardium, coronary vasospasm, direct catecholamine receptor-mediated effects and microvascular dysfunction. ${ }^{1}$ Reversible cerebral ischaemia with focal neurologic deficits is a rare manifestation of PY. ${ }^{3}$ In patients with PY, spasms of coronary, peripheral and cerebral arteries may occur from excessive catecholamine production. ${ }^{3}$ Cerebral artery spasm may result in either transient impairment of circulation or infarction. ${ }^{3}$ Hypertension can also cause cerebral infarction. ${ }^{3}$

Zhang et al in 2017 reported 163 cases of PY-associated cardiomyopathy. ${ }^{4}$ Hypertension was the commonest presenting feature and only $4 \%$ of patients had the classic triad of headaches, hypertension and diaphoresis. ${ }^{4}$ Agarwal et al noted that $47.4 \%$ of PY TC patients had a past medical history significant for hypertension. ${ }^{2}$ Biochemical confirmation of PY can be performed using 24-hour urine collection for catecholamines, metanephrines, (sensitivity $87.5 \%$; specificity $99.7 \%$ ) or plasma metanephrines (sensitivity $96 \%$; specificity $85 \%$ ). Localisation of the tumour using imaging studies (CT or MRI) can be done after biochemical confirmation. Meta-iodobenzyl guanidine scintigraphy may be used when these techniques fail to localise the tumour. Resection of PY led to improvement of cardiomyopathy in $94 \%$ while lack of resection was associated with death or cardiac transplantation in $44 \% .{ }^{4}$

In this patient, the time of diagnosis was estimated at about 2 years after the first report of signs of PY. This was similar to the time of diagnosis reported by Batisse et al. ${ }^{5}$ The diagnosis of PY related cardiomyopathies is often delayed in many cases. Chronic catecholamine exposure leads to myocardial fibrosis, low recovery rates and poorer prognosis. ${ }^{1}$ It is of utmost importance

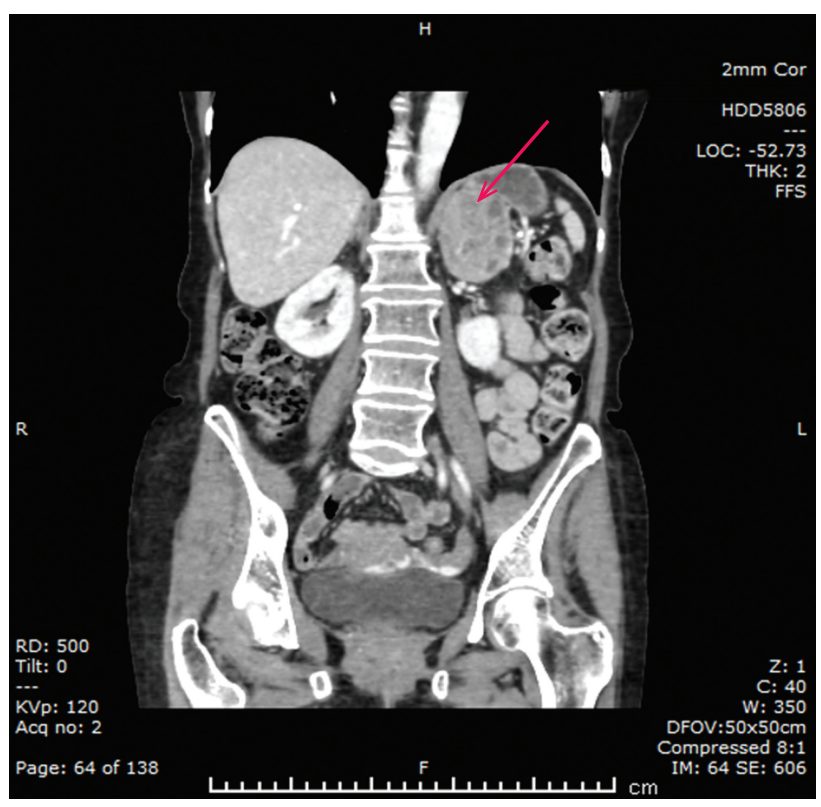

Fig 1. Computed tomography of the abdomen and pelvis showing left adrenal mass.

to recognise PY as a cause of cardiomyopathy. A diagnosis of unexplained cardiomyopathy should lead clinicians to a specific search for PY. If diagnosis is overlooked, consequences can be disastrous, even fatal; however, if PY is found, it is potentially curable. Early intervention (resection) may prevent progression to irreversible myocardial remodelling and mortality.

\section{Learning points}

> For the cardiologist, transient typical LV apical akinesis or dyskinesis on $\mathrm{ECHO}$, with absence of significant stenosis on coronary angiography should prompt consideration of Takotsubo cardiomyopathy. In selected patients, this should lead to an active search for a PY including measurement of blood or urinary catecholamines as all the classical clinical features of PY may not be present at the initial presentation. > For the endocrinologist, the diagnosis of a PY should lead to cardiac imaging (ECHO or MRI) in order to detect a potential silent cardiomyopathy. This will shorten the delay in introducing heart failure-specific drugs as well as improve perioperative management.

> For the stroke / transient ischaemic attack clinic specialists, in patients with suggestive history and typical ECHO findings, PY should be considered as a risk factor. Since our patient presented with various systemic symptoms to different specialists, recognition of this rare but potentially curable condition ought to be considered and collaborative working is crucial.

\section{References}

1 Afana M, Panchal RJ, Simon RM et al. Pheochromocytoma induced Takotsubo cardiomyopathy. Tex Heart Inst ] 2019;46:124-7. 
2 Agarwal V, Kant G, Hans N et al. Takotsubo-like cardiomyopathy in pheochromocytoma. Int J Cardiol 2011;153:241-8.

3 Lin PC, Hsu JT, Chung CM et al. Pheochromocytoma underlying hypertension, stroke, and dilated cardiomyopathy. Tex Heart Inst ] 2007;34:244-6.

4 Zhang R, Gupta D, Albert SG. Pheochromocytoma as a reversible cause of cardiomyopathy: Analysis and review of the literature. Int J Cardiol 2017;249:319-23.
5 Batisse-Lignier M, Pereira B, Motreff P et al. Acute and chronic pheochromocytoma-induced cardiomyopathies: different prognoses? A systematic analytical review. Medicine (Baltimore) 2015;94:e2198.

Address for correspondence: Dr Oluwatosin Kayode,

Withybush General Hospital, Fishguard Road, Haverfordwest, Pembrokeshire SA61 2PZ, UK.

Email: tosinod2001@yahoo.co.uk

\section{Your decisions change lives for good.}

\section{That's why, should something go wrong, we're with you every step of the way.}

24-hour advice line were speaking to a medico-legal adviser within 20 seconds. 